\title{
PROGRAMMING AND WIRING OF A LOW-COST TACHOMETER
}

\author{
László Rónai \\ assistant lecturer, University of Miskolc, Robert Bosch Department of Mechatronics \\ 3515 Miskolc, Miskolc-Egyetemváros, e-mail: ronai.laszlo@uni-miskolc.hu
}

\begin{abstract}
This paper deals with development of a measurement system, which is capable to calculate the rotational speed of a rotor. The unit contains a microcontroller, an infrared reflective optical sensor with resistors. The selection of current limiting resistors of the optical sensor will be discussed in order to set up an electrical circuit. The simulation of the electrical circuit was performed to compare the voltage and current values with the calculated ones. Microcontroller programs were written in Clanguage in order to compute the average frequency or the Root Mean Square (RMS) values of a rotating element. Comparative measurements were also carried out to check the accuracy of the developed system.
\end{abstract}

Keywords: microcontroller, RPM measurement, optical sensor

\section{Introduction}

Practical measurements may be needed not only in industry but even at home to make checking operation. For rotating elements, one of the most important parameters is the Revolutions Per Minute (RPM). Although tachometers are available on the market, they are usually a bit expensive [1], or do not provide a set of measurement data for postprocessing.

The word tachometer is derived from ancient Greek words takhys and metron, which mean quick and measure, respectively [2]. The tachometer can measure rotational speed of any rotating elements [3].

There are several methods to measure RPM according to [2]. Magnetic tachometers use the Hall effect phenomenon to detect the number of rotations, e.g., at bike speedometers. Mechanical tachometer contains two masses attached to the rotating shaft. These masses will have displacements in radial direction due to the centrifugal force of the rotating element. Electrodynamic tachometers, e.g., inductive pulse tachometer have no contact with measured rotating shaft, they contain a gear and a permanent magnet with a sensing coil [2]. Optical pulse tachometers generate square wave output signal, which frequency is proportional to the speed of the rotation [3]. The output types of a tachometer can be voltage, frequency value, or visual indication [2].

The main purpose of this paper is to create a circuit and program codes of a low-cost tachometer, which is capable to precisely determine the rotational speed of a shaft. In order to set up a test bench, a microcontroller (MCU) and an infrared optical sensor are required.

This paper is organized as follows: Section 2 deals with the designing process, simulation of the electrical circuit. Two program codes are developed in order to determine the frequency values of a rotating disc in laboratory conditions. Section 3 discusses the results of the comparative measurements with a Pepperl+Fuchs brand optical sensor. The concluding remarks will be given in Section 4. 


\section{The measurement system}

The system contains an ATmega328 microcontroller, which is wired to an Arduino Nano prototyping platform. A CNY70 reflective optical sensor [4] is used in order to measure the rotational speed of a rotor. Section 2.1 deals with the designing process of the electrical circuit, the developed $\mathrm{C}$ program codes are discussed in Section 2.2.

\subsection{The electrical circuit}

The CNY70 optical sensor consists a phototransistor and an infrared emitter LED, which has $\lambda=$ $950 \mathrm{~nm}$ wavelength. The package has a filter to block the visible light, and the typical operating range is between 0-5 mm. Two current limiting resistors should be placed into the circuit to protect the LED and set the allowable collector current.

The microcontroller operates $5 \mathrm{~V}$ TTL voltage, when the infrared LED forward current is $I_{F}=$ $30 \mathrm{~mA}$ the forward voltage is approximately $V_{F}=1,2 \mathrm{~V}$ (see Figure 1a). The current limiting resistor of the infrared LED can be calculated by using Ohm's law:

$$
R_{1}=\frac{V_{C C}-V_{F}}{I_{F}}=126.67 \Omega,
$$

where $V_{C C}$ is the supply voltage. A standard $R_{1}=120 \Omega$ resistor is used, in this case the forward current will be $I_{F} \cong 32 \mathrm{~mA}$. It is noted that the maximum allowable forward current is $I_{F, \max }=50 \mathrm{~mA}$ [4].
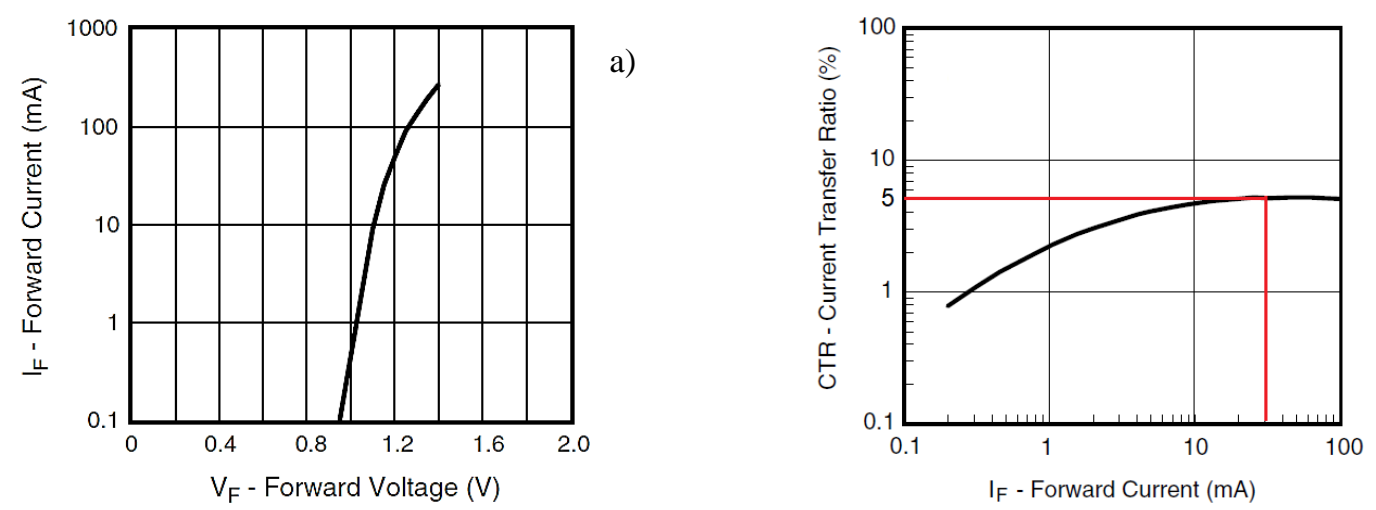

b)

Figure 1. a) Forward current - forward voltage, b) CTR - forward current diagrams [4]

The current transfer ratio (CTR) of the optical sensor can be determined by using the value of $I_{F}=$ $32 \mathrm{~mA}$ (see Figure 1b). The datasheet of the sensor notes that the diagram was determined with a Kodak neutral card, which is $0.3 \mathrm{~mm}$ far from the head of the sensor, and the $V_{C E}$ collector-emitter voltage of the phototransistor was $5 \mathrm{~V}$.

The value of the CTR is $5 \%$, which means the ratio between the $I_{F}$ forward current and the $I_{C}$ collector current is 0.05 . Therefore, the collector current is $I_{C}=0.05 \cdot 32 \mathrm{~mA}=1.6 \mathrm{~mA}$. The collector current will only be $1.6 \mathrm{~mA}$ if the object is very close to the phototransistor. In the [4] there is an example for the collector current in test condition: $V_{C E}=5 \mathrm{~V}$ and $I_{F}=20 \mathrm{~mA}$ (see Figure 2). The collector current will decrease as the distance increases. 
The maximum value of the collector current limiting resistor can be obtained by using dark current $I_{C E O}=200 n A$ [4] and the $V_{I H}$ high logic level voltage of the ATmega328, according to [5] the $V_{I H, \min }=0.6 \cdot V_{C C}=3 \mathrm{~V}$ :

$$
R_{2, \max }=\frac{V_{C C}-V_{I H}}{I_{C E O}}=10 \mathrm{M} \Omega .
$$

The dark current is a small current, which occurs when no photons enter to a photosensitive device.

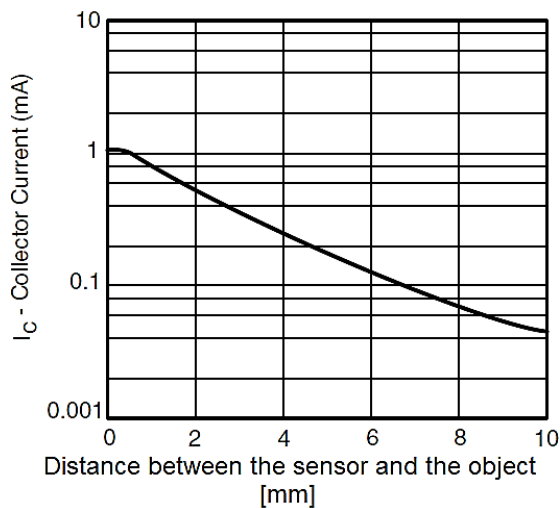

Figure 2. The collector current depends on the distance of the object [4]

According to Figure 2, in order to extend the distance range of the sensor, the $R_{2}$ collector current limiting resistor should be selected to ensure the nearly $5 \mathrm{~V}$ voltage drop across it at about $I_{C}=0.05 \mathrm{~mA}$.

In the course of the measuring process a digital input of the microcontroller will detect the signal logic levels. The logic 0 level can be provided by a

$$
R_{2}=\frac{V_{C C}}{I_{C}}=\frac{5 \mathrm{~V}}{0.05 \mathrm{~mA}}=100 \mathrm{k} \Omega
$$

current limiting resistor. In (3) the voltage-drop across the collector-emitter $\left(V_{C E}\right)$ is neglected. It is noted that if the collector-emitter voltage is below $1 \mathrm{~V}$, the $I_{C}$ current and the CTR will be also decrease [4]. Furthermore, when $R_{2}$ is large, the response time of the switching will increase.

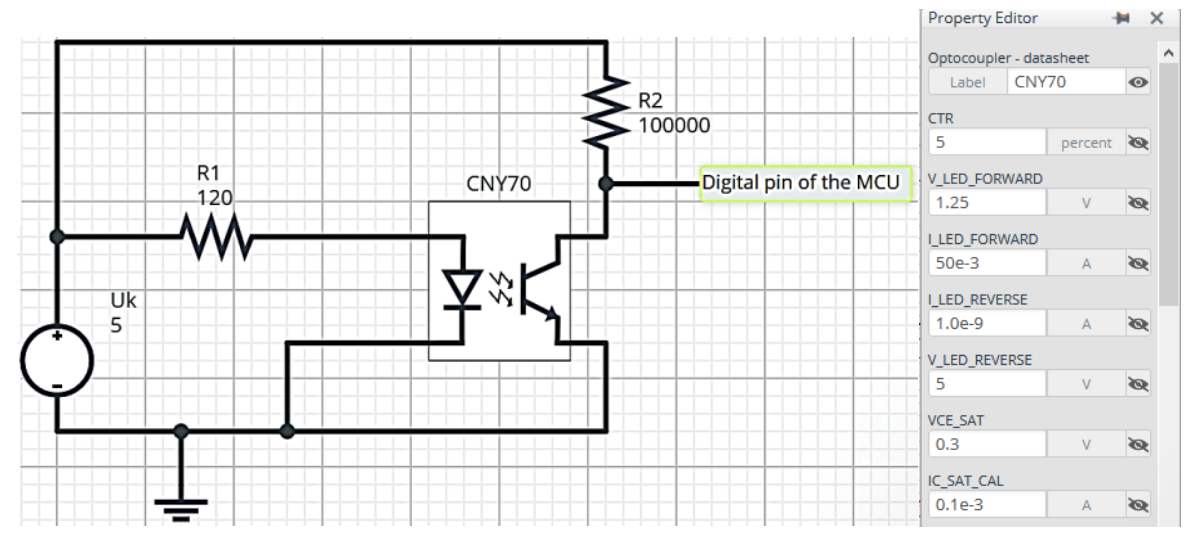

Figure 3. Electrical schematic of the system 
The electrical circuit is shown in Figure 3. The schematic was built in SystemVision ${ }^{\circledR}$ Cloud software online platform. The important data of the CNY70 sensor according to [4] can be given in this interface in order to simulate the voltages and currents.

The results of the simulation are shown in Figure 4. When the object is very close to the surface of the reflective sensor $V_{C E}=145.6 \mathrm{mV}$, and the average $I_{C}$ is $48.55 \mu \mathrm{A}$. Therefore, the voltage-drop of $R_{2}$ is $4.855 \mathrm{~V}$.

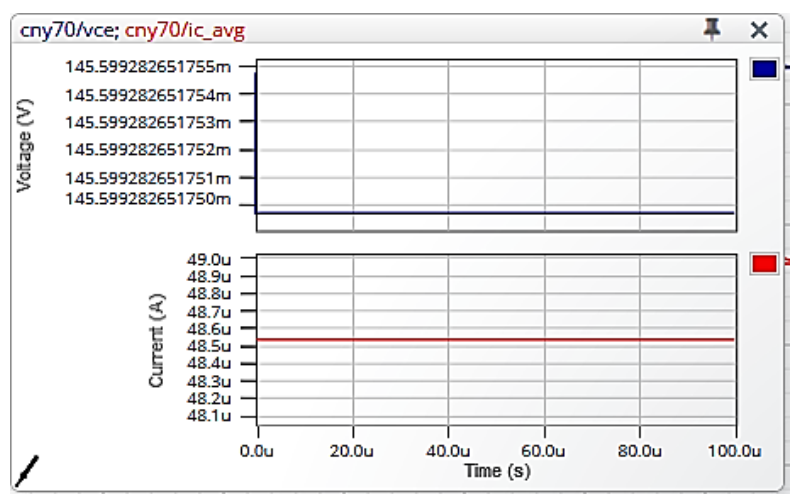

Figure 4. The simulation of the reflective sensor

The electrical circuit is placed onto a breadboard because it is easy to build up the connections between the electrical elements. The circuit can be seen in Figure 5.

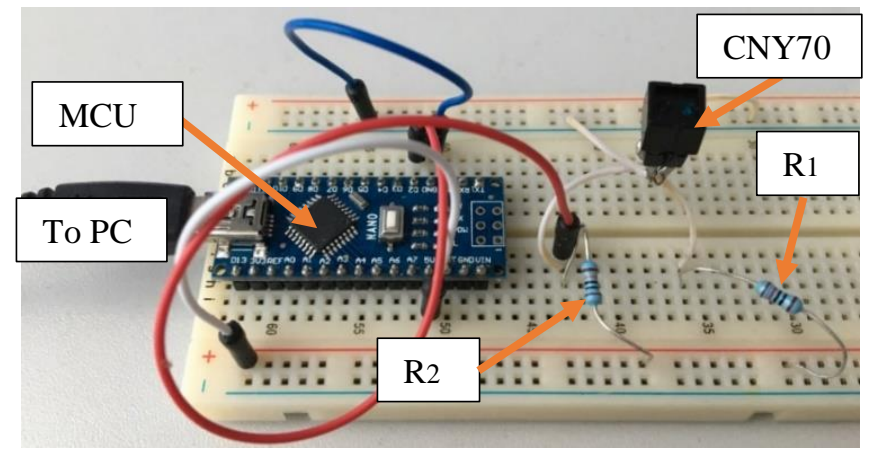

Figure 5. The assembled electrical circuit

It is noted that the use of the sensor in this configuration requires indoor conditions, preferably without other light sources. The maximum sensing distance is approximately $30 \mathrm{~mm}$ with bright object surface properties.

A test measurement was performed with the use of the 10-bit resolution analogue-digital converter of the MCU to check the voltage-drop values of the collector-emitter. Different voltages on collectoremitter were measured without sensing any object, and with a sensed object from distance $d=0.3 \mathrm{~mm}$ : $V_{C E}=4.75 \mathrm{~V}$ and $V_{C E}=0.195 \mathrm{~V}$, respectively.

\subsection{The developed program codes}

In order to measure the rotational speed of a rotor, two $C$ program code have been developed under Arduino IDE environment. The programs contain an Interrupt Service Routine (ISR), which is capable 
to use to count the revolutions. These types of routines can be triggered by external events. In this case FALLING option is used, which means that a logical 1 to logical 0 change will trigger the interrupt function. The ATmega 328 microcontroller has two external interrupt capabilities. The reflective sensor is wired with D2 digital input pin of the MCU. The variable, which is used in the main program code and the interrupt function should be defined as volatile.

The first program code, which computes the average frequency value for a desired time interval is shown in Table 1.

Table 1. The calculation of the average frequency

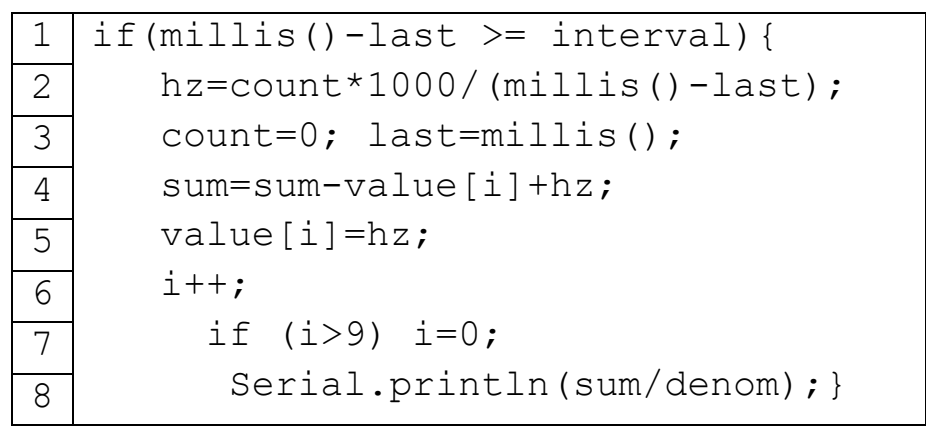

Between rows $1-8$ there is an if statement, which computes the average frequency of a rotor. The variable interval is set to $1 \mathrm{sec}$, if the elapsed time (millis()-last) is greater than or equal to this time, the actual frequency of the rotor is calculated (see row 2) by the use of variable count, which is in the ISR to count the rotation of an element. Thereafter in row 3, the variable count is equal to zero, and variable last gets the value of millis(). The function millis() provides the elapsed time value in milliseconds, which is measured from the $\mathrm{ON}$ state of the MCU.

In row 4, there is a command to produce a summation of the actual elements of array value. After that the $\mathrm{i}^{\text {th }}$ element of array value is equal to the actual measured frequency $(h z)$. Row 6 contains the postfix form of the index variable in order to be incremented at the next execution of the if statement. Another if statement is defined in row 7, since array value has ten elements. The data printing to the serial port can be found in row 8 , where the variable denom is the number of the array value.

With the use of the first program code the averaging process of the measured frequencies can be performed in a predefined time interval. The variable interval can be decreased if faster operation is required.

The second program code deals with the computation of the Root Mean Square (RMS) [6] value of a given time interval:

$$
R M S=\sqrt{\frac{\left(v_{1}^{2}+v_{1}^{2}+v_{1}^{2}+\cdots+v_{n_{a}}^{2}\right)}{n_{a}}}=\sqrt{\frac{\sum_{i=1}^{n_{a}\left(v_{i}^{2}\right)}}{n_{a}}},
$$

where $n_{a}$ is the size of the defined array, $v_{i}^{2}$ is the square of the actual measured frequency.

The structure of the second program is quite the same as in the first one. The definition of the time interval is the same as in Table 1. Instead of summation, the sum of squares is calculated and divided by the size of the array $\left(n_{a}\right)$. The square root operation is performed in the Serial.println() function. 


\section{Test measurements}

Test measurements were performed to compare the unit with an OJ500-M1K-E23 Pepperl+Fuchs optical sensor, which is equipped with fibre-optic cable. The rotation of a metal segmented disc is measured. The disc has four different coloured segments of which two has brighter surface. The supply voltage of the OJ500 optical sensor, and the drive motor is $24 \mathrm{~V} \mathrm{DC}$. A digital multimeter is used in order to determine the frequency of the square wave signal of the OJ500 optical sensor.

The test bench of the system is shown in Figure 6. A laptop is used to supply the MCU and receive the serial data. The bottom of the monitor of the laptop shows the latest frequency measurement. The upper part of the segmented disc is measured by the Pepperl+Fuchs optical sensor, and the CNY70 sensor is aligned to the lower part of the disc. Due to the two bright segments the measured frequencies are two time higher than the real one.

The measured frequency values with the CNY70 and the OJ500 sensor at the maximum adjustable rotating speed of the disc are shown in Table 2.

Table 2. The interval of the measured average frequencies at the maximum adjustable rotating speed

\begin{tabular}{|l|c|c||c|}
\cline { 2 - 4 } \multicolumn{1}{c|}{} & CNY70 & OJ500 & Difference \\
\hline Min. frequency & $236.5 \mathrm{~Hz}$ & $236.8 \mathrm{~Hz}$ & $0.3 \mathrm{~Hz}$ \\
\hline Max. frequency & $237.7 \mathrm{~Hz}$ & $238.1 \mathrm{~Hz}$ & $0.4 \mathrm{~Hz}$ \\
\hline Discrepancies (max-min) & $1.2 \mathrm{~Hz}$ & $1.3 \mathrm{~Hz}$ & \multicolumn{1}{|c}{} \\
\hline
\end{tabular}

Since, there are two bright segments on the disc, the measured frequencies must be divided by 2 . The real RPM of the system is between $7095-7143 \mathrm{rev} / \mathrm{min}$. According to the measured frequencies, the accuracy of the developed system is below $0.5 \mathrm{~Hz}$.

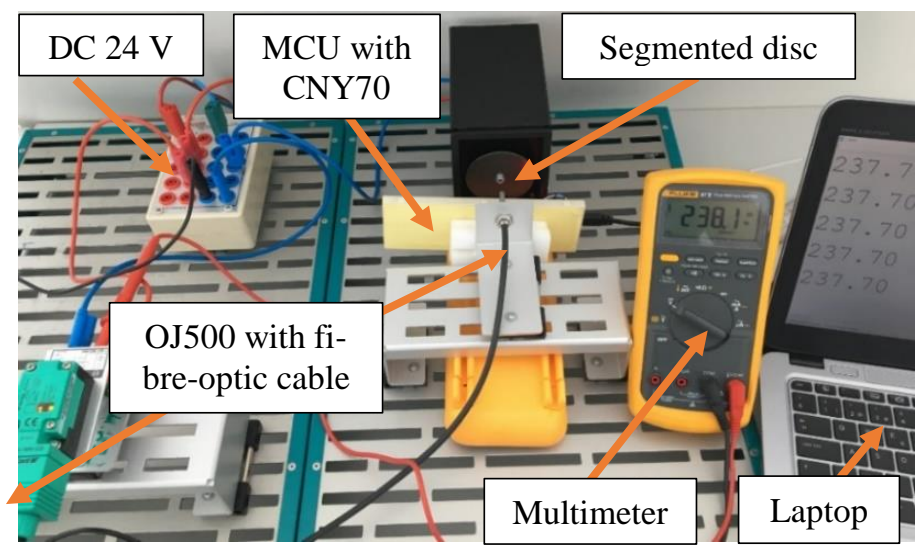

Figure 6. The test bench

With the use of the second program code the value RMS of the measured frequencies in different rotational speeds can be produced (see Figure 7ab). The measured frequency intervals at the minimum adjustable rotational speed are the following: $f_{C N Y 70}=30.3-30.59 \mathrm{~Hz}$ and $f_{O J 500}=30.33-$ $30.6 \mathrm{~Hz}$. It can be seen that the discrepancies between the CNY70 and the Pepperl+Fuchs optical sensor at lower rotational speed are smaller $\left(f_{\text {diff }}<0.1 \mathrm{~Hz}\right)$ than higher speeds.

The RMS determination method of the digital multimeter, e.g., the sampling time is unknown, therefore the measurement discrepancy may have resulted from this. 

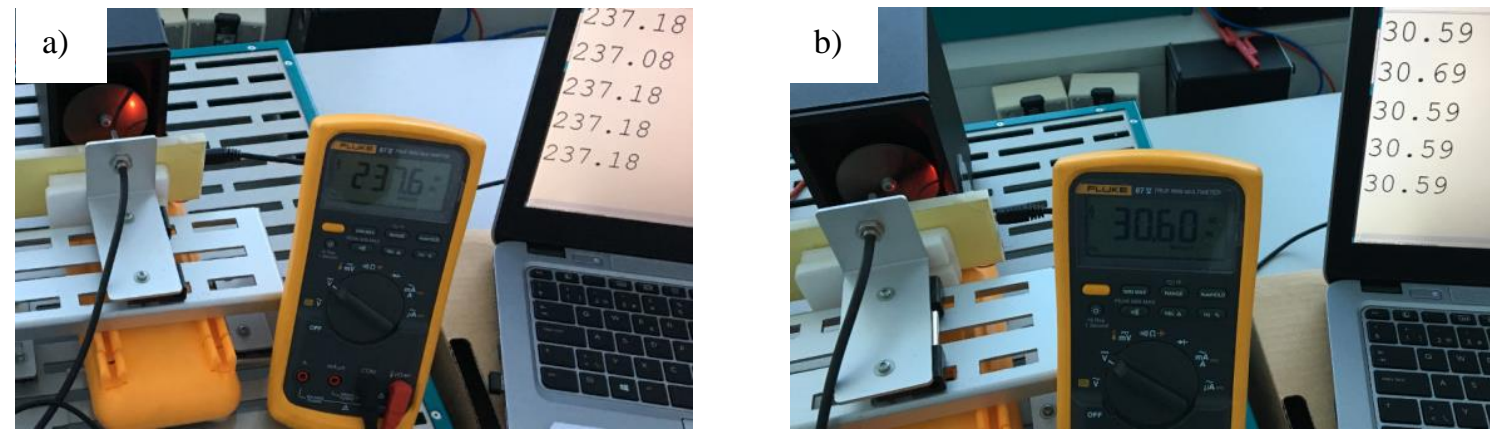

Figure 7. Computation of the RMS value a) at the minimum, b) at the maximum adjustable rotational speed

\section{Summary}

This paper was dealt with a low-cost system, which is capable to measure the rotational speed. The selection of the current limiting resistors of the optical sensor has been discussed. The simulation of the designed electrical circuit was performed with SystemVision ${ }^{\circledR}$ Cloud online software. The characteristic voltages, and currents were determined.

Comparative measurements were performed in order to determine the accuracy of the developed system. According to the measurement results, the accuracy of the system is within $0.5 \mathrm{~Hz}$. The OJ500 optical sensor has maximum $1.5 \mathrm{kHz}$ switching frequency, the developed system will capable to measure RPM values with higher measuring range. The price of the developed system is much lower than the commercially available OJ500 sensor.

\section{Acknowledgements}

The research reported here was carried out as part of the EFOP-3.6.1-16-2016-00011 "Younger and Renewing University - Innovative Knowledge City - Institutional development of the University of Miskolc aiming at intelligent specialization" project implemented in the framework of the Szechenyi 2020 program. The realization of this project is supported by the European Union, co-financed by the European Social Fund.

\section{References}

[1] Extech Instruments (2013). User Guide - Model RPM 33, FLIR Systems Inc.

[2] Dyer, S. A.: Wiley Survey of Instrumentation and Measurement, Wiley-IEEE Press, 2004, ISBN: 978-0-471-22165-4

[3] Ghosh, A. K.: Introduction to Measurements and Instrumentation, Motilal UK Books of India, 2009, ISBN 978-8120338586

[4] Vishay (2019). Reflective Optical Sensor with Transistor Output, datasheet, 83751, Vishay Intertechnology Inc.

[5] Atmel (2009). 8-bit AVR Microcontroller with 4/8/16/32K Bytes In-System Programmable Flash, Datasheet, Rev. 8025I-AVR-02/09.

[6] Jones, A. R.: Probability, Statistics and Other Frightening Stuff, Taylor \& Francis Group, 2019, ISBN 978-1-315-16006-1 https://doi.org/10.4324/9781315160061-1 